\title{
JUMPING TO A UNIFORM UPPER BOUND
}

\author{
HAROLD HODES
}

\begin{abstract}
A uniform upper bound on a class of Turing degrees is the Turing degree of a function which parametrizes the collection of all functions whose degree is in the given class. I prove that if $\underline{a}$ is a uniform upper bound on an ideal of degrees then $\underline{a}$ is the jump of a degree $\underline{c}$ with this additional property: there is a uniform bound $\underline{b}<\underline{a}$ so that $\underline{b} \vee \underline{c}<\underline{a}$.
\end{abstract}

Fix a recursive pairing function $(x, y) \mapsto\langle x, y\rangle$ from $\omega \times \omega$ onto $\omega$. For $f \in{ }^{\omega} \omega$, let $(f)_{x}(y)=f(\langle x, y\rangle)$; for $\mathscr{F} \subseteq{ }^{\omega} \omega, f$ parametrizes $\mathscr{F}$ iff $\mathscr{F}=\left\{(f)_{x} \mid x \in \omega\right\}$. Let $\leqslant_{T}$ and $\equiv_{T}$ be Turing reducibility and Turing equivalence on ${ }^{\omega} \omega$; a degree is an equivalence class under $\equiv_{T}$. Where $I$ is a set of degrees, a degree $\underline{a}$ is a uniform upper bound (u.u.b.) on $I$ iff some $f \in \underline{a}$ parametrizes $U I$. $I$ is an ideal iff $I$ is downward closed and closed under join; $I$ is a jump ideal iff it is also closed under jump. Let $I$ be a countable jump ideal and $\underline{a}$ be an upper bound on $I$. What can we say about degrees which jump to $\underline{a}$ ? Since $\underline{o}^{\prime} \in I$, Friedberg's theorem [2, p. 265] provides such a $\underline{b}$; but $\underline{b}$ is peculiar in that $\underline{b}^{\prime}=\underline{o}^{\prime} \vee \underline{b}$. Since $\underline{o}^{(2)} \in I$, we can relativize to $\underline{o}^{\prime}$ and obtain $\underline{b} \geqslant \underline{o^{\prime}}$; but now $b^{\prime}=\underline{o}^{(2)} \vee \underline{b}$. We would like to have $\underline{c} \vee \underline{b}<\underline{b}^{\prime}=\underline{a}$ for all $\underline{c} \in I$. In this note we show that if $\underline{a}$ is a u.u.b. on $I$, we can do this and more.

THEOREM. If $\underline{a}$ is $a$ u.u.b. on I there are $\underline{b}$ and $\underline{c}, \underline{c}$ a u.u.b. on I and $\underline{c} \vee \underline{b}<\underline{b}^{\prime}=\underline{a}$.

We prove this by using the trick of [3] within a construction like that used in [1, Theorem 3]. Fix $f \in \underline{a}, A \in \underline{a}, f$ parametrizing $\cup I$ and $A \in^{\omega} 2$. Where $K$ is a sequence $\left\langle n_{0}, \ldots, n_{l-1}\right\rangle ; \hat{K}=\left\langle(f)_{n_{0}}, \ldots,(f)_{n_{l-1}}\right\rangle$. If $u=\left\langle g_{0}, \ldots, g_{l-1}\right\rangle$ is a sequence of functions in ${ }^{\omega} \omega, \hat{u}$ is the partial function given by $\hat{u}(\langle i, x\rangle)=g_{i}(x)$ for $i<l ; u^{\vee}$ is the total extension of $\hat{u}$ such that $u^{\vee}(\langle i, x\rangle)=0$ for $i \geqslant 0$. We force with the language of arithmetic supplemented by the uninterpreted function symbol ' $g$ ' and predicate ' $\underline{B}$ '. A condition is a pair $\langle K, T\rangle$, where $K \in \omega^{<\omega}$ and $T$ is a total recursive perfect tree represented by its Gödel number. $\langle K, T\rangle \Vdash g(\underline{x})=y$ iff $(x)_{0}<\operatorname{lh}(K)$ and $(f)_{(x)_{0}}\left((x)_{1}\right)=y ;\langle K, T\rangle \Vdash \underline{B}(\underline{x})$ iff $x<\operatorname{lh} T(\langle\rangle)$ and $T(\langle\overline{)})(x)$ $=1$. A sequence $\delta$ is compatible with $K$ iff $\hat{K} \cup \delta$ is a function (viewing $\delta$ as a function on $\operatorname{lh}(\delta)$ ); $K$ floods $\delta$ iff $\delta \subseteq \hat{K}$. Note that if $\delta$ is compatible with $K$, some extension of $K$ floods $\delta$. Recall Sasso's starred subtrees: $T^{*}(\delta)=T\left(\delta^{*}\right)$ where $\delta^{*}=\left\langle(\delta)_{0}, 0,(\delta)_{1}, \ldots,(\delta)_{\mathrm{lh}(\delta)-1}, 0\right\rangle$ for $\delta \in$ Str.

Received by the editors August 21, 1981.

1980 Mathematics Subject Classification. Primary 03D30, 03D55.

Key words and phrases. Turing, degree, jump, ideal, uniform upper bound, tree.

(C)1982 American Mathematical Society 0002-9939/82/0000-0224/\$01.75 
Here is how we would like our construction to proceed. $K_{0}=\langle\rangle, T_{0}=\mathrm{id} \uparrow$ Str. Suppose we have $\left\langle K_{2 j}, T_{2 j}\right\rangle$. Stage $2 j+1$ : Fix $z=z\left(T_{2 j}\right)$, depending uniformly on (the Gödel number of) $T_{2 j}$ such that for all $B \in\left[T_{2 j}\right],\{z\}^{B}(z) \downarrow$ iff $B \notin\left[T_{2 j}^{*}\right]$.

Case 1. There are $\delta \in \operatorname{Str}$ and $\rho$ a finite sequence compatible with $K_{2 j}$ so that $\{s\}^{T_{2 j}\left(\delta^{*}\right) \oplus\left(\hat{K}_{2 j} \cup \rho\right)}(z)=0$. Let $\left\langle\delta_{2 j}, \rho\right\rangle$ be the least such. Let $K_{2 j+1}$ be the least extension of $K_{2 j}$ flooding $\rho$. Let $T_{2 j+1}(\tau)=T_{2 j}\left(\delta_{2 j}^{* n}\langle 1,1\rangle^{\cap} \tau\right)$.

Case 2. Otherwise. Let $K_{2 j+1}=K_{2 j}$ and $T_{2 j+1}=T_{2 j}^{*}$.

Stage $2 j+2 . K_{2 j+2}=K_{2 j+1}{ }^{n}\langle j\rangle$. Case 1. There is a $\delta \in \operatorname{Str}$ so that $\{j\}^{T_{2 j+1}(\delta)}(j) \downarrow$. Let $\delta_{2 j+2}$ be the least such $\delta$. Case 2. Otherwise; let $\delta_{2 j+2}=\langle\rangle$. Let $T_{2 j+2}(\tau)=T_{2 j+1}\left(\delta_{2 j+2}{ }^{n}\langle A(j)\rangle^{\cap} \tau\right)$ for all $\tau \in$ Str.

Let $g=\cup_{i} \hat{K}_{i} ; B=\bigcap_{i}\left[T_{i}\right]$. Clearly $g$ parametrizes $\cup I$. We selected $T_{2 j+1}$ to meet the requirement $B^{\prime} \neq\{j\}^{B \oplus g}$. For if Case 1 obtained at stage $2 j+1, B \notin\left[T_{2 j}^{*}\right]$; so $\{z\}^{B}(z) \downarrow$, so $B^{\prime}(z)=1$; but we have chosen $K_{2 j+1}$ to make sure that $\{j\}^{B \oplus g}(z)$ $=0$. If Case 2 obtained, $B \in\left[T_{2 j}^{*}\right]$; so $\{z\}^{B}(z) \uparrow$; so $B^{\prime}(z)=0$; but either $\{j\}^{B \oplus g}(z) \uparrow$ or it converges to something different from 0 . To compute $A$ from $B^{\prime}$ we must recover $\left\langle T_{s}\right\rangle_{s \in \omega}$ recursively in $B^{\prime}$, as a sequence of Gödel numbers. Suppose we have $T_{2 j}$. We have arranged to have $B$ signal to $B^{\prime}$ the choice of case and the value of $\delta_{2 j+1}$ in Case 1. For $B^{\prime}$ can tell whether $B \in\left[T_{2 j}^{*}\right]$. If not, we find the longest $\delta$ such that $T_{2 j}\left(\delta^{*}\right)$ is an initial segment of $B$; this is $\delta_{2 j+1}$; we can now obtain $T_{2 j+1}$. If $B \in\left[T_{2 j}^{*}\right.$ ], we know that $T_{2 j+1}=T_{2 j}$. An oracle for $B$ and $0^{\prime}$, which $B^{\prime}$ provides, suffices for carrying out even steps, that is, obtaining $\delta_{2 j+2}$; from this we get $A(j)=i$ iff $T_{2 j+1}\left(\delta_{2 j+2} \cap\langle i\rangle\right)$ is compatible with $B$; so we recover $A(j)$, and thus get $T_{2 j+2}$. Furthermore $B^{\prime}$ is recursive in the entire construction. The only hitch is that the construction just described is not recursive in $f$. For at stage $2 j+1$ we needed to answer a $\Sigma_{1}^{0}$ question about $K_{2 j}$.

Fortunately, since $f$ is a parametrization of $\cup I$ (and not just $\cup I \cap^{\omega} 2$ ), we can guess at an $m$ such that $(f)_{m}=\left(\hat{K}_{2 j}^{\vee}\right)^{\prime}$, so that from a certain point on our guesses settle and are right. So we modify the previous construction using the guessing technique of [1]. Working on requirement $B^{\prime} \neq\{j\}^{B \oplus g}$, we may guess that we are in Case 2, when actually we are in Case 1; so we may end up with $B^{\prime}(z)=1$ and, contrary to our intentions, $\{j\}^{B \oplus g}(z)=1$, where $z=z(T)$ and $T$ is the tree which we thought would meet the requirement. But then we shall just go back and attack that requirement again. At the end of stage $s$ we shall have $K_{0}^{s}, \ldots, K_{d(s)}^{s}$, our guesses at $K_{0}, \ldots, K_{d(s)}$, a tree $T_{s}$ (no guessing here!), a sequence $\rho_{s}: s \rightarrow \omega$ which is the portion of $g$ to which we are definitely committed, and a number $h(s)$ which tells us how far we have searched for witnesses to being in Case 1 on odd conditions. For $2 j+1<d(s), K_{2 j+1}^{s}$ was instituted by an attack on the requirement $B^{\prime}=\{j\}^{B \oplus g}$; the stage at which this attack occurred was $t(j, s) \leqslant s$. Let $c(j, T, K, \tau, q)=1$ iff our $q$ th guess at $\left(\hat{K}^{\vee}\right)^{\prime}$, says that there are $\delta \in \operatorname{Str}$ and $\rho$ compatible with $K$ and with $\tau$ such that $\{j\}^{T\left(\delta^{*}\right) \oplus(\hat{K} \cup \rho)}(z(T))=0 . c(j, T, K, \tau, q)=2$ otherwise. (Here $\tau$ is a finite sequence of numbers.)

Let $K_{0}^{0}=\langle\rangle, \rho_{0}=\varnothing, h(0)=0, d(0)=0, T_{0}=$ id Str. We describe stage $s+1$. Suppose we have $K_{0}^{s}, \ldots, K_{d(s)}^{s}, \rho_{s}, h(s), T_{s}, d(s)=2 j_{0} .2 j+1<d(s)$ is bad at 
$(s, r)$ iff $c\left(j, T_{t(j, s)-1}, K_{2 j}^{s}, \rho_{t(j, s)-1}, h(t(j, s))\right)=2$ and $c\left(j, T_{t(j, s)-1}, K_{2 j}^{s}, \rho_{t(j, s)-1}\right.$, $h(s)+r+1)=1$. In other words, when we instituted $K_{2 j}^{s}$ at stage $t(j, s)$ we thought we were in Case 2, but our $h(s)+r+1$ st guess at $\left(\hat{K}_{w j}^{s \vee}\right)^{\prime}$ says that we were in Case 1. Search for the least $r$ such that for some $j \leqslant j_{0}$ :

all $2 j^{\prime}+1<2 j+1$ are nonbad at $(s, r)$;

if $j<j_{0}, 2 j+1$ is bad at $(s, r)$;

if no $2 j+1<d(s)$ is bad at $(s, r), j=j_{0}$;

if $c\left(j, T_{s}, K_{2 j}^{s}, \rho_{s}, h(s)+r+1\right)=1$, then for some $\delta$ and $\rho$ which witness this fact, $\langle\delta, \rho\rangle \leqslant h(s)+r$.

There is such an $r$, and the least one determines a unique such $j$. Let $h(s+1)=h(s)$ $+r+1, d(s+1)=2 j+2, K_{i}^{s+1}=K_{i}^{s}$ for all $i \leqslant 2 j$. We now attack $B^{\prime} \neq\{j\}^{B \oplus g}$.

Case 1. $c\left(j, T_{s}, K_{2 j}^{s+1}, \rho_{s}, h(s+1)\right)=1$. Fix the least $\langle\delta, \rho\rangle$ so that $\delta$ and $\rho$ witness this fact. Let $K_{2 j+1}^{s+1}$ be the least extension of $K_{2 j}^{s+1}$ flooding $\rho$ and $\rho_{s}$; let $K_{2 j+2}^{s+1}=K_{2 j+1}^{s+1} \cap^{\cap}\langle j\rangle$. Let $T_{s+1}^{-}(\tau)=T_{s}\left(\delta_{s+1}^{*}{ }^{\cap}\langle 1,1\rangle^{\cap} \tau\right)$ for all $\tau \in$ Str, where $\delta=$ $\delta_{s+1}$.

Case 2. $c\left(j, T_{s}, K_{2 j}^{s+1}, \rho_{s}, h(s+1)\right)=2$. Let $K_{2 j+1}^{s+1}=K_{2 j}^{s+1}, K_{2 j+2}^{s+1}=K_{2 j+1}^{s+1} \cap\langle j\rangle$; let $T_{s+1}^{-}=T_{s}^{*}$. In either case, let $\rho_{s+1}$ be the least extension of $\rho_{s}$ compatible with $K_{2 j+2}^{s+1}$. If there is a $\delta^{\prime} \in \operatorname{Str}$ such that $\{s\}^{T_{s+1}^{-}\left(\delta^{\prime}\right)}(s) \downarrow$, let $\delta_{s+1}^{\prime}$ be the least such; otherwise $\delta_{s+1}^{\prime}=\langle\rangle . T_{s+1}(\tau)=T_{s+1}^{-}\left(\delta_{s+1}^{\prime} n^{n}\langle A(s)\rangle^{\cap} \tau\right)$ for all $\tau \in$ Str.

LEMMA. For any $j$ there is a stage $s(j)$ such that for all $s \geqslant s(j)$ and all $j^{\prime} \leqslant 2 j$ : $K_{j^{\prime}}^{s}=K_{j^{\prime}}^{s(j)}$, and no requirement $B^{\prime} \neq\left\{j^{\prime}\right\}^{B \oplus g}$ for $j^{\prime}<j$ is attacked at stage $s$.

Proof of this lemma is routine. Let $K_{j}=K_{j}^{s(j)}, g=\cup_{j} \hat{K}_{j}$. Note that $g=\lim _{s} \rho_{s}$. If $s+1$ is the last stage at which $B^{\prime} \neq\{j\}^{B \oplus g}$ is attacked, for no later $s^{\prime}$ is $j$ bad at $\left(s^{\prime}, 0\right)$; so the requirement is met. As before, $\left\langle T_{s}\right\rangle_{s \in \omega}$ is recursive uniformly in $B^{\prime}$, and $B^{\prime}$ can compute $A$, with $\delta_{s+1}$ and $\delta_{s+1}$ replacing the $\delta_{2 j+1}$ and $\delta_{2 j+2}^{\prime}$ of our previous attempt. The entire construction is recursive in $f$, since construction of $T_{s+1}$ from $T_{s+1}^{-}$requires only an oracle for $0^{\prime}$, which $f$ provides, so $B^{\prime} \leqslant_{T} f$. Q.E.D.

In general, for what sorts of upper bounds $\underline{a}$ on $I$ are there $\underline{b}$ so that for all $\underline{c} \in I$, $\underline{b} \vee \underline{c}<\underline{b}^{\prime}=\underline{a}$ ? More pressing, however, is the problem: is every u.u.b. on $I$ (or, for that matter, on the ideal of arithmetic degrees) the jump of an u.b. on $I$ ?

\section{REFERENCES}

1. H. Hodes, More on uniform upper bounds, J. Symbolic Logic (to appear).

2. H. Rogers, The theory of recursive functions and effective computability, McGraw-Hill, New York, 1967.

3. L. Sasso, A minimal degree not realizing least possible jump, J. Symbolic Logic 39 (1974).

Department of Philosophy, Cornell University, Ithaca, New York 14853

Current address: Department of Philosophy, Tufts University, Medford, Massachusetts 02155 